\title{
Effectiveness of a modified Mediterranean diet recipe plus moderate exercise before and during pregnancy among women at high risk of gestational diabetes
}

\author{
Fang $\mathrm{Liu}^{1}$, Zhaohui $\mathrm{Hu}^{1}$, Yinfang Dong ${ }^{1}, \mathrm{Ke} \mathrm{Han}^{1}$, and Xiaoyan Ding ${ }^{1}$ \\ ${ }^{1}$ Zibo Central Hospital
}

March 18, 2021

\begin{abstract}
Background: Women with gestational diabetes mellitus (GDM) have elevated risk of type 2 diabetes mellitus (T2DM). Several studies have indicated healthy lifestyle reduces the prevalence of GDM. However, limited evidence shows the association between Mediterranean diet (MD) and GDM incidence in China. The aim of the current study was to establish a Chinese-habit-based MD recipe and investigate the effects of the modified MD on GDM. This trial recruited gestational age women who were planning a pregnancy in the near future with at least one risk factor for GDM. Methods: These participants were randomly assigned into the MD group and control group. They were required to follow diet interventions at least 6 months prior to pregnancy until delivery. Average dietary intake, glucose and insulin metabolism in 26-28-week of gestation, maternal and neonatal outcomes in each group were analyzed to assess the effects of MD. We analyzed the clinical outcomes of 580 participants, 294 in the MD group and 286 in the control group. Results: The MD group had a high intake of protein, vitamins, and dietary fibers, whereas low intake of fat, resulting in improved insulin and glucose metabolism. Meanwhile, women and their newborns in the MD group showed a reduced proportion of complications. Conclusion: The modified MD intervention started before pregnancy shows a preventive effect on GDM, also benefits the mother and their newborns in other outcomes.
\end{abstract}

Effectiveness of a modified Mediterranean diet recipe plus moderate exercise before and during pregnancy among women at high risk of gestational diabetes

Fang Liu, Zhaohui Hu, Yinfang Dong, Ke Han, Xiaoyan Ding*

Department of Obstetrics, ZIBO Central Hospital, No. 54 Gongqingtuan West Road, Zhangdian District, Zibo 255036, Shandong, China

${ }^{*}$ Corresponding author

Xiaoyan Ding

Department of Obstetrics, ZIBO Central Hospital, No. 54 Gongqingtuan West Road, Zhangdian District, Zibo 255036, Shandong, China

Email: sdzbdingxiaoyan@163.com

Tel: 86-18678186121

Running title: Modified Mediterranean diet recipe plus

What is already known about this topic?

Women with gestational diabetes mellitus (GDM) have elevated risk of type 2 diabetes mellitus (T2DM). 
What does this article add?

The MD group had a high intake of protein, vitamins, and dietary fibers, whereas low intake of fat, resulting in improved insulin and glucose metabolism.

\section{Abstract}

Background: Women with gestational diabetes mellitus (GDM) have elevated risk of type 2 diabetes mellitus (T2DM). Several studies have indicated healthy lifestyle reduces the prevalence of GDM. However, limited evidence shows the association between Mediterranean diet (MD) and GDM incidence in China. The aim of the current study was to establish a Chinese-habit-based MD recipe and investigate the effects of the modified MD on GDM. This trial recruited gestational age women who were planning a pregnancy in the near future with at least one risk factor for GDM.

Methods: These participants were randomly assigned into the MD group and control group. They were required to follow diet interventions at least 6 months prior to pregnancy until delivery. Average dietary intake, glucose and insulin metabolism in 26-28-week of gestation, maternal and neonatal outcomes in each group were analyzed to assess the effects of MD. We analyzed the clinical outcomes of 580 participants, 294 in the MD group and 286 in the control group.

Results: The MD group had a high intake of protein, vitamins, and dietary fibers, whereas low intake of fat, resulting in improved insulin and glucose metabolism. Meanwhile, women and their newborns in the MD group showed a reduced proportion of complications.

Conclusion: The modified MD intervention started before pregnancy shows a preventive effect on GDM, also benefits the mother and their newborns in other outcomes.

Keywords: Gestational diabetes mellitus (GDM); Mediterranean-like diet; diet intervention; improved outcomes

\section{Introduction}

Gestational diabetes mellitus (GDM) means "glucose intolerance that begins or is first diagnosed during pregnancy" 1. GDM is manifested in not only glucose intolerance but also insulin resistance. In recent years, GDM has become one of the most serious risks for pregnant women, and the prevalence of GDM is increasing all over the world. It was reported that GDM is associated with various obstetric complications, including hydramnios and preterm delivery ${ }^{2}$. and several adverse outcomes in newborns, such as congenital malformation and neonatal respiratory distress syndrome ${ }^{3}$. In addition, women with a history of GDM are at an increased risk of type 2 diabetes mellitus (T2DM) in later life, they are also exposed to a high risk of GDM relapse when they become pregnant again ${ }^{4,5}$. However, with the thorough liberalization of two-child policy in China, more Chinese women tend to have a second child, including those who had GDM before. To help women release the burden of GDM during their pregnancy, it is important to establish a new lifestyle, especially a healthy diet recipe in accordance with traditional Chinses eating habits.

Nowadays, a Mediterranean-style diet is regarded as a healthy eating habit, which is wildly adopted in Greece, Spain, France and other southern European countries along the Mediterranean coast. A Mediterranean diet (MD) refers to high intake of vegetables, fruits, fish, nuts and olive oil, while low intake of red meat and sugary drinks ${ }^{6}$. Mounting evidence has demonstrated that keeping a MD effectively reduces the risk of various chronic diseases, such as cancer, cardiovascular disease and obesity ${ }^{7-9}$. Nevertheless, in China with a high incidence rate of GDM at 21.9\%, few studies were conducted on the effectiveness of the MD on GDM before and during pregnancy.

In this study, we aimed to access whether the MD has a control effect on GDM among women aged between 18 and 50 years old with at least one risk factor for GDM who were planning a pregnancy in the near future.

\section{Materials and methods}

Study design 
The current randomized clinical trial adopted the per-protocol analysis, and was conducted to lessen the potential confounding effects ${ }^{10}$. Women were recruited between 20 December 2016 and 15 June 2019 through advertising, telephone inquiring and health care consulting. The eligible participants with matched physical condition were assigned to two test groups. The study was approved by ZIBO Central Hospital and registered in ZIBO Central Hospital.

\section{Inclusion criteria}

Women were eligible to enter the clinical trial if they met the following three conditions: at high risk for GDM; planning for pregnancy in the near future; of the childbearing age between 18 and 50 years old. If the participants had at least one of the following risk factors: body mass index (BMI) exceeds $25 \mathrm{~kg} / \mathrm{m}^{2}$; a history of GDM; any signs of obesity in their family; diagnosis of polycystic ovary syndrome (PCOS) before pregnancy, they were regarded as high-risk cohorts. All participants were required to provide informed written consent before they were randomly assigned into either the intervention group or the control group. To ensure objectivity, randomized assignment was performed by an experienced clinical staff utilizing computer generated random numbers, and researchers were blind to the allocation results until they finished the final analyses.

The intervention started immediately when the participants were assigned into the test groups. To accurately analyze the effects of improved MD on GDM, a longer time was needed in this clinical trial since the diet intervention was a long process. To meet this purpose, we believed that the participants should receive at least 6 months of intervention before their pregnancy ${ }^{11}$. Therefore, women who become pregnant within 6 months were excluded. Furthermore, women who had any of the following items were excluded at the beginning of the trial: under the age of 18; unmarried; smoking; alcohol drinking; being pregnant at the time of enrollment; suffering from other types of diseases, such as urolithiasis, kidney disease, thyroid diseases or gastrointestinal diseases; HIV, Hep-B or Hep-C positive patients; diagnosed with diabetes before randomization assignment. In addition, women who had taken illegal drugs or other insulin interfering drugs within 6 months before pregnancy ${ }^{12}$, and those who did not agree to sign the informed consent for certain reasons were also excluded.

Intervention

The intervention was based on a MD and continued from randomized assignment to delivery. All the participants were requested to take a comprehensive physical examination before the randomized assignment, in order to ensure the balance and accuracy of the assignment. From the beginning of intervention, recruited women were required to take a physical examination every 4 weeks at the same medical center. Once participants became pregnant, they were asked to follow physical examinations at least a) once in the first 3 months; b) once every month in the middle 3 months; c) once every two weeks during 28 to 36 gestation weeks; d) once every week after 36 weeks, until they completed a safe delivery.

The traditional MD prefers to cook with extra virgin olive oil and recommends to keep low intake of red meat and processed meat ${ }^{13}$, which is difficult for Chinese women to follow completely during the intervention. To solve this problem, we established a nutrition support team, containing 5 nutritionists and 3 obstetricians, to propose an improved Mediterranean-like diet, which is better adapted for Chinese women. The nutrition support team delivered the intervention over a personalized one-to-one session at the beginning of the intervention. Since olive oil was not widely accepted in China, participants were allowed to use the ordinary rapeseed oil as a substitute for olive oil, but with an allocated amount of no more than $0.3 \mathrm{~L}$ per week. In addition, given the fact that Chinese women show a higher potential for mild anemia and hypotension ${ }^{14}$, a moderate consumption of red meat per day was recommended. Moreover, alcohol drinking was inappropriate for women during pre-pregnancy or pregnancy. Taken together, we formulated an adapted intervention plan based on the MD, including: replacing olive oil with rapeseed oil, soybean oil, or peanut oil, and the daily intake was controlled at lower than $30 \mathrm{~g}$; keeping a daily consumption of red meat at 50-70 g; and alcohol drinking was prohibited. The improved MD recipe was more suitable for Chinese women and improved the compliance of this trial.

For their thorough understanding of the MD, participants were requested to join in a training class after 
being assigned to test groups. The training class lasted 40-60 min, and all participants were provided with MD guidance when they finished the training. The diet intervention officially started from this face-to-face consulting. In the middle of the intervention, we followed up with the women every few days through phone calls or Wechat messages to reinforce the dietary goals. Furthermore, our nutritionists provided the latest information about the MD and recipe guidance during the trial. When the participants had any questions or suggestions about the intervention plan, they could communicate with their nutritionists or doctors through phone calls or Wechat at any time.

Participants in the control group received routine nutrition guidance and health care, but no dietary requirements. All participants were requested not to adjust their daily physical activity, an exercise time no less than 30 minutes per day was recommended in both the intervention group and the control group.

Dietary assessment

We referred to the methodology of calculating MD scores to assess dietary conditions of the participants ${ }^{15}$. Briefly, daily servings of vegetables, fruits, legumes, cereals, fish, dairy products, and meat were recorded and scored. All participants recorded their daily food and physical activities using a phone app termed Nutritional Calculator. Then the individual's daily intake was calculated and averaged using the Nutritionist IV software ${ }^{16}$. The participants' MD adherence was evaluated once a month. According to the evaluation reports, we adjusted the diet recipe of each individual to ensure all the participants followed an accurate intervention.

Data collection

All the basic information of the recruited women was collected though a computerized questionnaire before they joined the clinical trial. To safeguard participant privacy, the information was only accessible to the professional administrators who performed data analysis. The information involved in the questionnaire were listed as follows:

* Socio-demographic data: age, marital status, number of children, and level of education.

* Physical condition: body weight, BMI, blood glucose, blood pressure, etc.

* Medical history of diseases: macrosomia in previous pregnancies, PCOS, earlier gestational glucose intolerance/GDM, family history of diabetes, mild anemia, hypotension, etc.

* Food consumption and dietary intake information: dietary preferences and appetite.

\section{Outcome}

In this study, the primary outcomes were the occurrence of women with GDM in 26-28 gestation weeks, the newborns' birthweight and neonatal Apgar score. GDM was diagnosed based on the oral glucose tolerance test (OGTT) in 26-28 gestation weeks ${ }^{11}$. Once the OGTT of participants varied abnormally, appropriate intervention and treatment would be guided by health care specialists. The quantitative insulin check index (QUICKI), homeostasis model of assessment of insulin resistance (HOMA-IR), and homeostasis model of assessment of beta cell function (HOMA-b) were detected to assess insulin resistance ${ }^{17}$. As for the neonatal outcome, the birthweight of newborns was recorded. The newborn whose birthweight exceeds $4000 \mathrm{~g}$ was defined as macrosomia, while those less than $2500 \mathrm{~g}$ were defined as underweight ${ }^{18}$.

Secondary outcome was weight gain during pregnancy, which was calculated on the last measured weight before parturition and the weight measured at the beginning of the gestation. According to the guidelines of Institute of Medicine, excessive weight gain is defined as follows: $>18 \mathrm{~kg}$ for underweight; $>16 \mathrm{~kg}$ for normal weight; $>11.5 \mathrm{~kg}$ for overweight; and $>9 \mathrm{~kg}$ for obese. The definition was based on the initial BMI of participants. Moreover, preterm delivery, natural childbirth or cesarean delivery, pre-eclampsia after 20 weeks of gestation, and blood pressure were also considered as secondary outcomes.

Sample Size 
Initially, we used a randomized clinical trial calculation formula to design the sample size, in which the risk $\AA=0.05$, risk $\beta=0.2$, and a rate of $20 \%$ for follow-up lose. According to a previous study ${ }^{11}$, the incidence of GDM was $40 \%$ under usual prenatal care, whereas the intervention was supposed to reduce the proportion. The statistical power of the study was 0.8 . On the basis of the assumption, a total of 749 eligible women were recruited in the clinical trial. Except for 169 dropouts in allocation and follow-up process, data from 580 participants were analyzed, with 294 in the MD group and 286 in the control group.

\section{Statistical Analysis}

The data were analyzed according to the purpose of the intervention trial. The statistical analysis in the current study was performed utilizing SPSS, version 18. (SPSS Inc., Chicago, IL, USA). Data were expressed as mean (SD) unless otherwise stated. Continuous and nominal data were analyzed by Student $\mathrm{t}$ test and $\chi^{2}$-test, respectively. $P<0.05$ was considered as statistically significant.

\section{Results}

The flow chat illustrating the study design is shown in Figure 1. A total of 749 women were enrolled into our trial at the beginning. After assessing their baseline information for eligibility, 70 women were excluded, and the remaining 679 women were randomly assigned into the intervention (MD group, $\mathrm{n}=339$ ) or the control group $(n=340)$. During the course of the study, $\sim 15 \%$ of the participants were withdrawn from each group. The interference factors included: the participants did not conceive during the study; the participants became pregnant within 6 months; the participants felt sickness in stomach because of the diet intervention, etc. (Fig. 1). In the end, 294 participants in the MD group and 286 in the control group were followed up and included in the analysis.

The baseline characteristics of the participants are summarized in Table 1. No significant differences were observed between the two groups in baseline characteristics, in terms of age [29.5(4.6) years in MD group, $30.3(4.5)$ years in Control group] and BMI before pregnancy [27.4(4.4) kg/m² in MD group, 27.5(3.7) kg/m² in control group], or BMI after randomization [Overweight 135(45.9\%) and obese 12(4.1\%) in MD group, Overweight 137(47.9\%) and obese 10(3.5\%) in control group]. In addition, percentage of primiparous [43.2\% in MD group, $39.5 \%$ in control group], proportion of macrosomia in previous pregnancies [4.1\% in MD group, $3.8 \%$ in control group], earlier GDM [36 in MD group, 34 in control group], family history of diabetes [111 in MD group, 107 in control group], and PCOS [9 in MD group, 8 in control group], as well as other socio-demographic data were also statistically the same between all participants in the two groups. The time to pregnancy was $8.9(2.8)$ and $8.7(3.2)$ weeks for MD group and control group, respectively. No significant difference was observed.

Nutritional differences in daily intakes between MD and routine diet.

Average dietary intakes of participants in the MD) group and control group are shown in Table 2. During the intervention, participants in the MD group were required to consume high intakes of vegetables, fruits, legumes, cereals, and fish, and low intake of processed meat and food rich in animal fat. Meanwhile, participants in the control group kept their normal eating habits that are common for Chinese: they took more red meat and refined grain in daily life. Table 2 shows that in comparison with control group, participants in MD group consumed more vegetables [558.25(147.69) g/day vs 449.43(132.63) g/day, p=0.008], legumes [443.61(257.44) g/day vs 435.47(249.81) g/day, p=0.032], fish [60.74(23.59) g/day vs $41.75(26.28) \mathrm{g} /$ day, $\mathrm{p}<0.001]$, and dairy products [468.43(176.72) g/day vs 316.25(189.69) g/day, $\mathrm{p}<0.001]$. On the contrary, the participants' average consumption of meat and derivatives in the control group was significantly higher than that in the MD group [65.35(27.42) g/day vs $57.92(24.66) \mathrm{g} /$ day, $\mathrm{p}<0.001]$. In terms of nutritional components, a MD is rich in dietary fibers and protein, but low in fat and energy. Therefore, participants in the MD group had significantly higher protein [(15.32(3.19) vs 12.71(3.27) (\% of total energy), $\mathrm{p}<0.001)$, dietary fibers $\left[(29.73(8.93)(\mathrm{g} / \mathrm{d})\right.$ vs $24.61(8.62)(\mathrm{g} / \mathrm{d}), \mathrm{p}=0.016)$, and $\mathrm{B}_{12}[(29.73(8.93)(\mathrm{g} / \mathrm{d})$ vs 24.61(8.62) $(\mu \mathrm{g} / \mathrm{d})$, $\mathrm{p}=0.016)$, whereas lower level of energy [1961.84(124.93) $(\mathrm{kcal} / \mathrm{d})$ vs 2042.63(116.67) $(\mathrm{kcal} / \mathrm{d}), \mathrm{p}=0.018]$ and fat $[20.45(7.44)$ vs $23.62(6.53)$ ( $\%$ of total energy), $\mathrm{p}=0.006]$. 
Glucose and insulin metabolism of the participants

Glucose and insulin metabolism of the participants were assessed by fasting blood glucose (FBG), HOMAIR, HOMA- $\beta$ and QUIKI (Table 3). All the evaluated indicators were detected at both baseline and 26-28 weeks of gestation, in order to demonstrate the variation of glucose intolerance and insulin resistance, as well as to diagnose GDM. From baseline to 26-28 gestational weeks, the FBG level of participants in the MD group remained unchanged [97.25(9.97) $(\mathrm{mg} / \mathrm{dL})$ vs $95.46(9.12)(\mathrm{mg} / \mathrm{dL}), \mathrm{p}=0.153]$, whereas it significantly increased in the control group [94.09(7.23) $(\mathrm{mg} / \mathrm{dL})$ vs106.37(8.29) $(\mathrm{mg} / \mathrm{dL}), \mathrm{p}=0.022]$. In addition, HOMAIR $[2.7(1.7)$ vs 2.5(1.3), $\mathrm{p}=0.232$ ] and HOMA- $\beta$ [46.7(17.4) vs 47.4(15.3), $\mathrm{p}=0.114$ ] stayed at the same level in the MD group, while slight increased levels were observed in the control group \{HOMA-IR [12.9(6.2) vs $15.9(6.9), \mathrm{p}=0.012]$, HOMA- $\beta$ [46.3(18.2) vs 55.1(19.7), $\mathrm{p}=0.017]\}$. Moreover, the QUICKI score was improved in the MD group [0.47(0.16) vs $0.49(0.15), \mathrm{p}=0.042]$, whereas it was decreased in the control group $[0.48(0.17)$ vs $0.36(0.18), \mathrm{p}=0.021]$. These data indicated that the diet intervention had a positive effect on glucose and insulin metabolism for pregnant women.

Other maternal and neonatal outcomes

Besides glucose and insulin metabolism, we also analyzed other maternal physical symptoms and neonatal outcomes. The final results are summarized in Table 4 . Both the proportions of women with GDM $[24.8 \%$ vs $33.9 \%, \mathrm{p}=0.013]$ and the proportion of participants with excessive gestational weight gain $[26.9 \% \mathrm{vs} 37.1 \%$, $\mathrm{p}=0.044]$ were markedly decreased in the MD group. Nevertheless, the type of delivery, percentage of preeclampsia [3.7\% vs $31 \%, \mathrm{p}=0.297$ ], and the preterm delivery proportion [ $6.1 \%$ vs $7 \%, \mathrm{p}=0.179]$ showed no differences between the two groups.

Among the newborns, the average birthweight in the two groups stayed in a comparable level, whereas the proportion of macrosomia was lower in the MD group than in the control group [3.4\% vs 4.2\%, $\mathrm{p}=0.038$ ]. Meanwhile, 5-min Apgar score was significantly higher in the MD group [10.6(0.7) vs 9.2(0.6), $\mathrm{p}=0,021]$. In addition, 1-min Apgar score and percentage of underweight newborn did not differ in the two groups.

\section{Discussion}

In recent years, the incidence of GDM is rising steadily. GDM was reported to be a risk factor for T2DM, and is also associated with various adverse outcomes, causing long-term sufferings for both the mothers and their newborns. However, there has been increasing evidence suggesting that healthy lifestyle intervention has the potential to benefit the women with GDM and/or T2DM. For instance, the American National Institutes of Health proposed the Diabetes Prevention program ${ }^{19}$, which means an individualized intervention of eating and physical activity, leading to a $50 \%$ decrease in diabetes incidence in the pre-diabetes cohorts. Similarly, GDM women followed 1 month of DASH (Dietary Approaches to Stop Hypertension) diet showed improved pregnancy outcomes ${ }^{20}$. Besides, women in western countries who complied with a healthy diet before pregnancy, such as MD, exhibited an ameliorative glucose tolerance and lower incidence of GDM during their pregnancy. In order to demonstrate the effect of a healthy diet on GDM among Chinese women, in this study, we provided an improved MD in accordance with traditional Chinese eating habits, and conducted an intervention trial before and during women's pregnancy.

The typical MD emphasizes on high intakes of fruits, vegetables, whole grains, legumes, nuts, and olive oil; moderate intake of fish and dairy products; low consumption of healthy fats, such as poultry; and limited intake of red meat. Also, there are other important components in traditional MD, including a glass of red wine and keeping proper physical activity. From the perspective of nutrition, the MD is rich in monoand poly-unsaturated fatty acids, protein, vitamins and dietary fibers ${ }^{21}$, which are beneficial for preventing many metabolic and chronic diseases. For example, Thaminda et $\mathrm{al}^{22}$. and $A$ semi $\mathrm{Z}^{23}$ found that the MD protected against vascular and coronary heart diseases. Moreover, Letenneur $L$ et al ${ }^{24}$ found that MD had a potential to decrease the risk of Alzheimar disease and cognitive decline. Artal $R$ et al ${ }^{25}$ confirmed that the low-energy and low-fat diet resulted in a dramatic reduction in the risk of T2DM and obesity.

Nevertheless, it is difficult for Chinese people, especially pregnant and pre-pregnant women, to entirely follow 
the typical MD. Because the traditional Chinese diet is composed in a large proportion of refined grains and red meat, which is quite different from the MD. And women who become pregnant or prepare to be pregnant should not drink alcohol. According to this situation, to better investigate whether MD intervention benefits GDM outcomes among Chinese women, we proposed a Chinese-habit-based Mediterranean-like diet in our current clinical study. We replaced olive oil with rapeseed oil, and added an appropriate consumption of red meat in the daily intake, due to Chinese women's high incidence of mild anemia and hypotension during their gestation. In addition, all the participants were recommended to take proper physical exercise every day. Meanwhile, other elements of MD remained unchanged except for alcohol consumption.

In the present trial, we analyzed clinical outcomes of 580 participants, 294 in the MD group and 286 in the control group. At the beginning of the diet intervention, no significant differences in baseline characteristics were observed between the two groups, including the levels of glucose intolerance and insulin resistance (Table 1\&3). During the intervention, the MD group consumed more vegetable, legumes, fish and dairy products, whereas less meat and derivatives. That helped participants obtain better protein, vitamins and dietary fibers but less fat (Table 2). As a result, when the participants entered their 26-28 weeks of gestation, the MD group showed improved insulin and glucose metabolism. But participants in the control group exhibited a worse glucose intolerance and insulin resistance, which associated with an increased incidence of GDM (Table 3\&4). These data indicated that our Mediterranean-like diet intervention started before pregnancy exhibited a preventive effect against GDM. Our results were consistent with an earlier study, which suggested that high adherence to the MD was related to declined GDM incidence in a dose and time dependent manner ${ }^{26}$.

Besides diabetic symptoms, we also evaluated other maternal outcomes and neonatal complications. As expected, women in the MD group had fewer cases of excessive gestational weight gain, and their newborns showed reduced proportion of macrosomia as well as higher 5-min Apgar score (Table 4). These data implied that the MD intervention improved not only the physical condition of gravidae but also that of their newborns. Consistently, Rogozińska $E$ et al. observed that MD intervention and physical activity lessened the caesarean section rates ${ }^{27}$. He, J.R and his colleagues' study suggested women followed MD presented better post-partum recovery and declined incidence of neonatal complications ${ }^{28}$. Therefore, we conclude that our MD intervention strongly ameliorates GDM among Chinese women, which also benefits both the mothers and their newborns in other pregnancy outcomes.

We used average daily nutritional intake to estimate adherence to the MD, while other studies used different methods, such as Trichopoulou index, and $24 \mathrm{~h}$ diet score ${ }^{29}$. Several studies demonstrated the effect of MD in women without GDM in their previous pregnancies ${ }^{30,31}$. Whereas in our study, we recruited women with a history of GDM into both the intervention and control group, then analyzed the GDM-preventing potential of MD. As for evaluating the diet quality, some study tended to separate the effects of each nutrient ${ }^{32}$. However, when assessing the results of a diet intervention, we should give priority to the synergistic effects of all the components. We therefore conclude that our MD intervention has a positive effect on GDM improvement, although a few dietary components differed between the two groups. In the future study, it is worth studying whether the Chinese-habit-based MD also has beneficial effects on T2DM, which may provide a new reference for clinical treatment of T2DM.

A common limitation of long-term clinical trials is participant-loss during the follow-up. To solve this problem, participants were encouraged to record their daily intake and physical activity using a phone app termed Nutritional Calculator, and professional administrators collected the participants' information every day from the data cloud. Furthermore, we used Wechat to keep constant contact with the participants and reminded them to keep recording, which facilitated the interaction between researchers and the participants. Since we expected an attrition rate of $20 \%$, it was rational that our overall follow-up rate was $85 \%$. However, another possible limitation is the deviation from MD. Although we adjusted the participants' MD recipe every month according to their records, women still tend to have their own preference for what they should consume. This was the major cause for inaccurate intervention, leading to biased dietary intake. Moreover, although we found a significant decline in some maternal outcomes, the type of delivery, the rates of preterm 
delivery and pre-eclampsia were not influenced by MD intervention. Finally, a further modification should be done in traditional MD diet before the intervention can be utilized to other diseases.

\section{Conclusion}

In conclusion, a Mediterranean-like diet before and during pregnancy had a preventive effect on GDM development, also improved several maternal and neonatal outcomes. For controlling the development of GDM, adherence to a Mediterranean-like diet prior to pregnancy should be promoted among all Chinese women.

\section{Competing interests}

The authors declare that they have no competing interests.

\section{Funding}

None.

\section{Acknowledgments}

None.

\section{References}

1. Buchanan TA, Xiang AH. Gestational diabetes mellitus. J Clin Invest. 2005;115(3):485-491.

2. Briana DD, Boutsikou M, Marmarinos A, Gourgiotis D, Malamitsi-Puchner A. Perinatal sclerostin concentrations in abnormal fetal growth: the impact of gestational diabetes. J Matern Fetal Neonatal Med.2019;32(13):2228-2232.

3. Huynh J, Xiong G, Bentley-Lewis R. A systematic review of metabolite profiling in gestational diabetes mellitus. Diabetologia.2014;57(12):2453-2464.

4. Rimar Z, Milas V, Medimurec M, Mesic I. Respiratory distress syndrome in newborns of gestational age of over 32 weeks. Coll Antropol.2014;38(2):621-626.

5. Shah BR, Retnakaran R, Booth GL. Increased risk of cardiovascular disease in young women following gestational diabetes mellitus.Diabetes Care. 2008;31(8):1668-1669.

6. Salas-Salvado J, Bullo M, Estruch R, et al. Prevention of diabetes with Mediterranean diets: a subgroup analysis of a randomized trial.Ann Intern Med. 2014;160(1):1-10.

7. Kesse-Guyot E, Ahluwalia N, Lassale C, Hercberg S, Fezeu L, Lairon D. Adherence to Mediterranean diet reduces the risk of metabolic syndrome: a 6-year prospective study. Nutr Metab Cardiovasc Dis.2013;23(7):677-683.

8. Estruch R, Ros E, Martinez-Gonzalez MA. Mediterranean diet for primary prevention of cardiovascular disease. N Engl J Med.2013;369(7):676-677.

9. Rodriguez-Bernal CL, Rebagliato M, Iniguez C, et al. Diet quality in early pregnancy and its effects on fetal growth outcomes: the Infancia y Medio Ambiente (Childhood and Environment) Mother and Child Cohort Study in Spain. Am J Clin Nutr. 2010;91(6):1659-1666.

10. Babadi M, Khorshidi A, Aghadavood E, et al. The Effects of Probiotic Supplementation on Genetic and Metabolic Profiles in Patients with Gestational Diabetes Mellitus: a Randomized, Double-Blind, PlaceboControlled Trial. Probiotics Antimicrob Proteins.2019;11(4):1227-1235.

11. Luoto R, Kinnunen TI, Aittasalo M, et al. Primary prevention of gestational diabetes mellitus and large-for-gestational-age newborns by lifestyle counseling: a cluster-randomized controlled trial. PLoS Med. 2011;8(5):e1001036. 
12. American Diabetes A. Diagnosis and classification of diabetes mellitus. Diabetes Care. 2010;33 Suppl 1:S62-69.

13. B HAW, Dodds J, Placzek A, et al. Mediterranean-style diet in pregnant women with metabolic risk factors (ESTEEM): A pragmatic multicentre randomised trial. PLoS Med. 2019;16(7):e1002857.

14. Polley BA, Wing RR, Sims CJ. Randomized controlled trial to prevent excessive weight gain in pregnant women. Int J Obes Relat Metab Disord. 2002;26(11):1494-1502.

15. Tognon G, Lissner L, Saebye D, Walker KZ, Heitmann BL. The Mediterranean diet in relation to mortality and CVD: a Danish cohort study. Br J Nutr. 2014;111(1):151-159.

16. Perez-Ferre N, Del Valle L, Torrejon MJ, et al. Diabetes mellitus and abnormal glucose tolerance development after gestational diabetes: A three-year, prospective, randomized, clinical-based, Mediterranean lifestyle interventional study with parallel groups. Clin Nutr.2015;34(4):579-585.

17. Zhang H, Su S, Yu X, Li Y. Dietary epigallocatechin 3-gallate supplement improves maternal and neonatal treatment outcome of gestational diabetes mellitus: a double-blind randomised controlled trial. $J$ Hum Nutr Diet. 2017;30(6):753-758.

18. McGowan CA, McAuliffe FM. The influence of maternal glycaemia and dietary glycaemic index on pregnancy outcome in healthy mothers. Br $J$ Nutr. 2010;104(2):153-159.

19. Chowdhury A, Sarkar J, Chakraborti T, Pramanik PK, Chakraborti S. Protective role of epigallocatechin3-gallate in health and disease: A perspective. Biomed Pharmacother. 2016;78:50-59.

20. Asemi Z, Samimi M, Tabassi Z, Esmaillzadeh A. The effect of DASH diet on pregnancy outcomes in gestational diabetes: a randomized controlled clinical trial. Eur J Clin Nutr. 2014;68(4):490-495.

21. Schwingshackl L, Hoffmann G. Adherence to Mediterranean diet and risk of cancer: an updated systematic review and meta-analysis of observational studies. Cancer Med. 2015;4(12):1933-1947.

22. Liyanage T, Ninomiya T, Wang A, et al. Effects of the Mediterranean Diet on Cardiovascular OutcomesA Systematic Review and Meta-Analysis.PLoS One. 2016;11(8):e0159252.

23. Asemi Z, Samimi M, Tabassi Z, Sabihi SS, Esmaillzadeh A. A randomized controlled clinical trial investigating the effect of DASH diet on insulin resistance, inflammation, and oxidative stress in gestational diabetes. Nutrition. 2013;29(4):619-624.

24. Letenneur L, Proust-Lima C, Le Gouge A, Dartigues JF, Barberger-Gateau P. Flavonoid intake and cognitive decline over a 10-year period. Am J Epidemiol. 2007;165(12):1364-1371.

25. Artal R, Catanzaro RB, Gavard JA, Mostello DJ, Friganza JC. A lifestyle intervention of weight-gain restriction: diet and exercise in obese women with gestational diabetes mellitus. Appl Physiol Nutr Metab. 2007;32(3):596-601.

26. Olmedo-Requena R, Gomez-Fernandez J, Amezcua-Prieto C, Mozas-Moreno J, Khan KS, JimenezMoleon JJ. Pre-Pregnancy Adherence to the Mediterranean Diet and Gestational Diabetes Mellitus: A Case-Control Study. Nutrients. 2019;11(5).

27. International Weight Management in Pregnancy Collaborative G. Effect of diet and physical activity based interventions in pregnancy on gestational weight gain and pregnancy outcomes: meta-analysis of individual participant data from randomised trials. BMJ.2017;358:j3119.

28. He JR, Yuan MY, Chen NN, et al. Maternal dietary patterns and gestational diabetes mellitus: a large prospective cohort study in China. Br J Nutr. 2015;113(8):1292-1300.

29. Tobias DK, Zhang C, Chavarro J, et al. Prepregnancy adherence to dietary patterns and lower risk of gestational diabetes mellitus.Am J Clin Nutr. 2012;96(2):289-295. 
30. Karamanos B, Thanopoulou A, Anastasiou E, et al. Relation of the Mediterranean diet with the incidence of gestational diabetes. Eur J Clin Nutr. 2014;68(1):8-13.

31. Roman-Vinas B, Ribas Barba L, Ngo J, Martinez-Gonzalez MA, Wijnhoven TM, Serra-Majem L. Validity of dietary patterns to assess nutrient intake adequacy. Br J Nutr. 2009;101 Suppl 2:S12-20.

32. Jacques PF, Tucker KL. Are dietary patterns useful for understanding the role of diet in chronic disease? Am J Clin Nutr.2001;73(1):1-2.

\section{Figure legends}

Figure 1. Flow diagram of the study design: participant selection, allocation and follow-up. MD group, Mediterranean Diet group.

Table 1. Baseline characteristics of the participants.

\begin{tabular}{|c|c|c|c|}
\hline Characteristics & MD group $(n=294)$ & Control group $(n=286)$ & $\mathrm{p}$ value \\
\hline Age (y) & $29.5(4.6)$ & $30.3(4.5)$ & 0.495 \\
\hline Primiparous, n (\%) & $127(43.2)$ & $113(39.5)$ & 0.586 \\
\hline Education, n (\%) & & & 0.354 \\
\hline High school or higher & $81(27.6)$ & $74(25.9)$ & \\
\hline Secondary & $134(45.6)$ & $126(44.1)$ & \\
\hline Primary school or lower & $79(26.8)$ & $86(30.0)$ & \\
\hline $\begin{array}{l}\text { BMI after } \\
\text { randomization, n (\%) }\end{array}$ & & & 0.457 \\
\hline $\begin{array}{l}\text { Overweight }(\mathrm{BMI}>25 \\
\left.\mathrm{kg} / \mathrm{m}^{2}\right)\end{array}$ & $135(45.9)$ & $137(47.9)$ & \\
\hline $\begin{array}{l}\text { Obese }(\text { BMI }>30 \\
\left.\mathrm{kg} / \mathrm{m}^{2}\right)\end{array}$ & $12(4.1)$ & $10(3.5)$ & \\
\hline BMI before pregnancy & $27.4(4.4)$ & $27.5(3.7)$ & 0.270 \\
\hline $\begin{array}{l}\text { Macrosomia in previous } \\
\text { pregnancies, } \mathrm{n}(\%)\end{array}$ & $12(4.1)$ & $11(3.8)$ & 0.299 \\
\hline $\begin{array}{l}\text { Earlier gestational } \\
\text { glucose } \\
\text { intolerance/GDM, n } \\
(\%)\end{array}$ & $36(12.2)$ & $34(11.9)$ & 0.232 \\
\hline $\begin{array}{l}\text { Family history of } \\
\text { diabetes, } \mathrm{n}(\%)\end{array}$ & $111(37.8)$ & $107(37.4)$ & 0.256 \\
\hline PCOS, n (\%) & $9(3.1)$ & $8(2.8)$ & 0.358 \\
\hline
\end{tabular}

PCOS, polycystic ovarian syndrome.

Data are expressed as mean (SD), unless otherwise indicated. We analyzed continuous and nominal data with Student $t$ test and $\chi^{2}$-test, respectively.

Values in bold indicate $\mathrm{p}<0.05$ of MD group vs Control group.

Table 2. Dietary intakes of participants.

\begin{tabular}{llll}
\hline & MD group $(\mathrm{n}=294)$ & Control group $(\mathrm{n}=286)$ & $\mathrm{p}$ value \\
\hline Vegetables (g/day) & $558.25(147.69)$ & $449.43(132.63)$ & $\mathbf{0 . 0 0 8}$ \\
Fruits (g/day) & $443.61(257.44)$ & $435.47(249.81)$ & 0.107 \\
Legumes (g/day) & $12.29(7.72)$ & $11.17(6.89)$ & $\mathbf{0 . 0 3 2}$ \\
Cereals (g/day) & $246.58(101.74)$ & $252.72(112.53)$ & 0.095
\end{tabular}




\begin{tabular}{llll}
\hline & MD group $(\mathrm{n}=294)$ & Control group $(\mathrm{n}=286)$ & $\mathrm{p}$ value \\
\hline Fish (g/day) & $60.74(23.59)$ & $41.75(26.28)$ & $<\mathbf{0 . 0 0 1}$ \\
Dairy products (g/day) & $468.43(176.72)$ & $316.25(189.69)$ & $<\mathbf{0 . 0 0 1}$ \\
Meat and derivatives (g/day) & $57.92(24.66)$ & $65.35(27.42)$ & $<\mathbf{0 . 0 0 1}$ \\
Energy (kcal/d) & $1961.84(124.93)$ & $2042.63(116.67)$ & $\mathbf{0 . 0 1 8}$ \\
Carbohydrate (\% of total energy) & $54.89(8.47)$ & $58.94(7.93)$ & 0.197 \\
Protein (\% of total energy) & $15.32(3.19)$ & $12.71(3.27)$ & $<0.001$ \\
Fat $(\%$ of total energy) & $20.45(7.44)$ & $23.62(6.53)$ & $\mathbf{0 . 0 0 6}$ \\
Iron $(\mathrm{mg} / \mathrm{d})$ & $13.64(3.32)$ & $12.81(3.07)$ & 0.064 \\
$\mathrm{~B}_{12}(\boldsymbol{\mu g} / \mathrm{d})$ & $5.45(3.66)$ & $4.63(4.13)$ & $\mathbf{0 . 0 4 3}$ \\
Dietary fibre $(\mathrm{g} / \mathrm{d})$ & $29.73(8.93)$ & $24.61(8.62)$ & $\mathbf{0 . 0 1 6}$ \\
\hline
\end{tabular}

Data are expressed as mean (SD). Student's t-test was used to analyze the normally distributed data, whereas a Mann-Whitney U-test was used to analyze the non-normally distributed data. Values in bold indicate $\mathrm{p}$ $<0.05$ of MD group vs Control group.

Table 3. Glucose and insulin metabolism of the participants at baseline and gestational 26-28 weeks

\begin{tabular}{lllll}
\hline Glucose metabolism & MD group $(\mathrm{n}=294)$ & MD group $(\mathrm{n}=294)$ & MD group $(\mathrm{n}=294)$ & Control group $(\mathrm{n}=286)$ \\
\hline & Baseline & $26-28 \mathrm{w}$ & $\mathrm{p}$ value & Baseline \\
Fasting blood glucose $(\mathrm{mg} / \mathrm{dL})$ & $97.25(9.97)$ & $95.46(9.12)$ & 0.153 & $94.09(7.23)$ \\
Insulin $(\mu \mathrm{IU} / \mathrm{mL})$ & $13.1(5.9)$ & $12.6(4.3)$ & 0.089 & $12.9(6.2)$ \\
HOMA-IR & $2.7(1.7)$ & $2.5(1.3)$ & 0.232 & $2.5(1.9)$ \\
HOMA- $\beta$ & $46.7(17.4)$ & $47.4(15.3)$ & 0.114 & $46.3(18.2)$ \\
QUICKI & $0.47(0.16)$ & $0.49(0.15)$ & $\mathbf{0 . 0 4 2}$ & $0.48(0.17)$ \\
\hline
\end{tabular}

HOMA-IR and HOMA- $\beta$, homeostasis model of assessment of insulin resistance and beta cell function; QUICKI, quantitative insulin check index.

Data are expressed as mean (SD). Student's t-test was used to analyse the normally distributed data, whereas a Mann-Whitney U-test was used to analyse the non-normally distributed data.

Values in bold indicate $\mathrm{p}<0.05$.

Table 4. Maternal and neonatal outcomes of the study.

\begin{tabular}{llll}
\hline Outcomes & MD group $(\mathrm{n}=294)$ & Control group $(\mathrm{n}=286)$ & $\mathrm{p}$ value \\
\hline Maternal & & & \\
GDM, n(\%) & $73(24.8)$ & $97(33.9)$ & $\mathbf{0 . 0 1 3}$ \\
$\begin{array}{l}\text { Gestational weight } \\
\text { gain, } \mathrm{kg}\end{array}$ & $11.9(3.7)$ & $13.7(4.6)$ & 0.058 \\
$\begin{array}{l}\text { Excessive gestational } \\
\text { weight gain, } \mathrm{n}(\%)\end{array}$ & $79(26.9)$ & $106(37.1)$ & $\mathbf{0 . 0 4 4}$ \\
$\begin{array}{l}\text { Preterm delivery, }<37 \\
\text { wk, } \mathrm{n}(\%)\end{array}$ & $18(6.1)$ & $20(7.0)$ & 0.179 \\
Type of delivery, n(\%) & & & \\
$\begin{array}{l}\text { Normal } \\
\text { Instrumental }\end{array}$ & $172(58.5)$ & $171(59.8)$ & 0.211 \\
Cesarean & $39(13.3)$ & $42(14.7)$ & \\
& $83(28.2)$ & $73(25.5)$ &
\end{tabular}




\begin{tabular}{llll}
\hline Outcomes & MD group $(\mathrm{n}=294)$ & Control group $(\mathrm{n}=286)$ & $\mathrm{p}$ value \\
\hline $\begin{array}{l}\text { Pre-eclampsia, } \mathrm{n}(\%) \\
\text { Neonatal }\end{array}$ & $11(3.7)$ & $9(3.1)$ & 0.297 \\
$\begin{array}{l}\text { Birthweight, } \mathrm{g} \\
\text { Adequate 2500-4000, }\end{array}$ & $3149(374)$ & & \\
$\mathrm{n}(\%)$ & $266(90.4)$ & $251(87.8)$ & 0.067 \\
Low $<2500, \mathrm{n}(\%)$ & $18(6.1)$ & & 0.107 \\
Macrosomia $>4000$, & $10(3.4)$ & $23(8.0)$ & \\
$\mathrm{n}(\%)$ & & $12(4.2)$ & 0.047 \\
1-min Apgar & $9.5(0.6)$ & $9.4(0.5)$ & $\mathbf{0 . 0 3 1}$ \\
5-min Apgar & $10.6(0.7)$ & $9.2(0.6)$ & 0.238 \\
\hline
\end{tabular}

Data are expressed as mean (SD), unless otherwise indicated. We analyzed continuous and nominal data

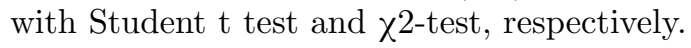

Values in bold indicate $\mathrm{p}<0.05$ of MD group vs Control group.

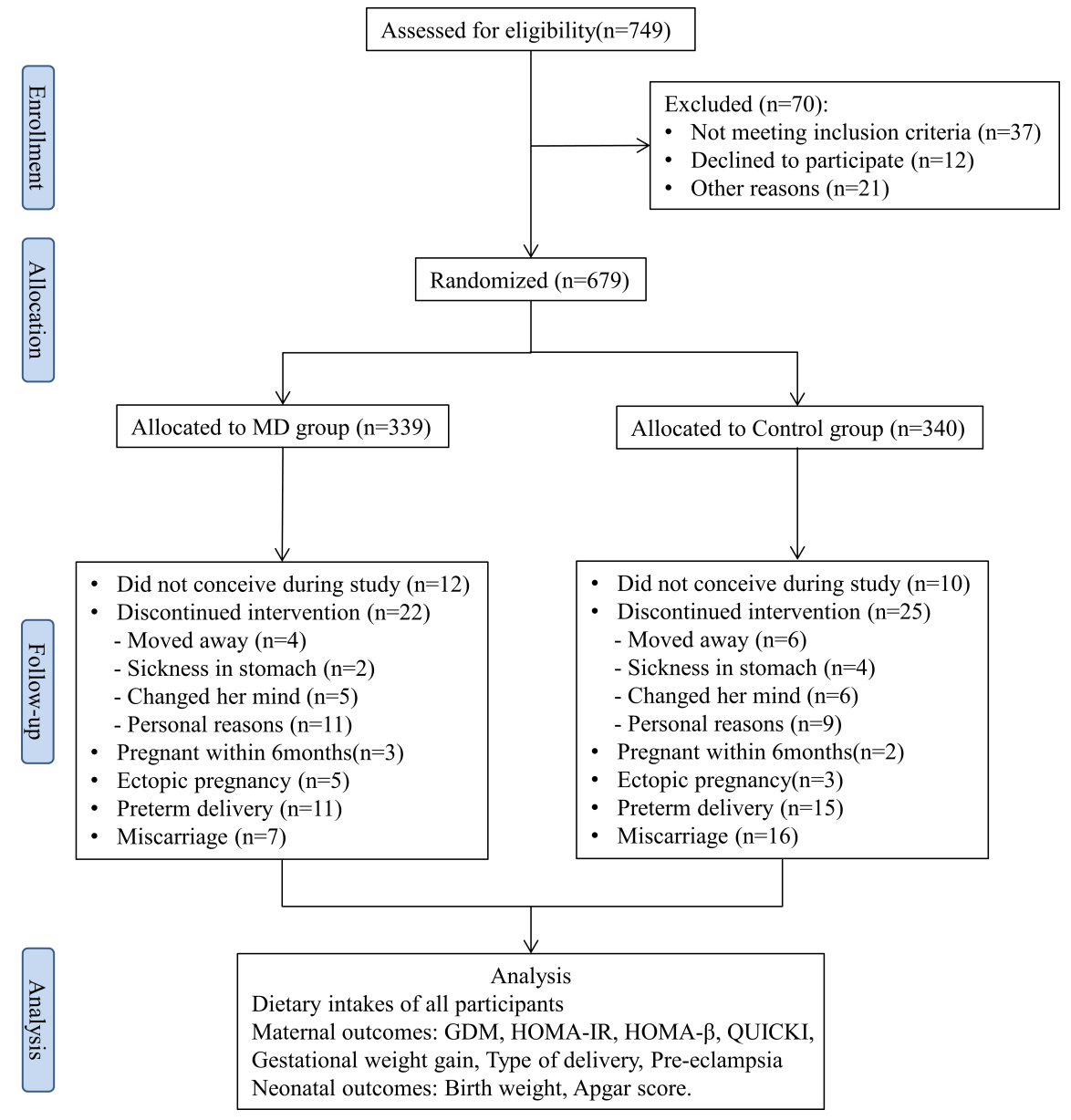

